\title{
Degradation kinetics and pathways of three calcium channel blockers under UV irradiation
}

\author{
Bing Zhu ${ }^{a}$, Bozo Zonja ${ }^{b}$, Oscar Gonzalez ${ }^{c}$, Carme Sans ${ }^{c}{ }^{*}$, Sandra Pérez ${ }^{b}$, \\ Damia Barceló ${ }^{\text {b, d }}$, Santiago Esplugas ${ }^{c}, \mathrm{Ke} \mathrm{Xu}^{\mathrm{a}}$, Zhimin Qiang ${ }^{\text {a, * }}$ \\ ${ }^{a}$ Key Laboratory of Drinking Water Science and Technology, Research Center for Eco-Environmental Sciences, Chinese Academy of Sciences, 18 Shuang-qing \\ Road, Beijing 100085, China \\ ${ }^{\mathrm{b}}$ Water and Soil Quality Research Group, Department of Environmental Chemistry, IDAEA-CSIC, 18-26 c/Jordi Girona, 08034 Barcelona, Spain \\ ${ }^{c}$ Department of Chemical Engineering, University of Barcelona, Marti i Franques 1, 08028 Barcelona, Spain \\ ${ }^{\mathrm{d}}$ Catalan Institute of Water Research, c/Emili Grahit, 101 Edifici H2O, Parc Cientific $i$ Tecnologic de la Universitat de Girona, E-17003 Girona, Spain
}

\section{A R T I C L E I N F O}

\section{Article history:}

Received 28 February 2015

Received in revised form 13 May 2015

Accepted 14 May 2015

Available online 16 May 2015

\section{Keywords:}

Calcium channel blockers

UV irradiation

Photolysis kinetics

Transformation byproducts

Wastewater treatment plant effluent

\begin{abstract}
A B S T R A C T
Calcium channel blockers (CCBs) are a group of pharmaceuticals widely prescribed to lower blood pressure and treat heart diseases. They have been frequently detected in wastewater treatment plant (WWTP) effluents and downstream river waters, thus inducing a potential risk to aquatic ecosystems. However, little is known about the behavior and fate of CCBs under UV irradiation, which has been adopted as a primary disinfection method for WWTP effluents. This study investigated the degradation kinetics and pathways of three commonly-used CCBs, including amlodipine (AML), diltiazem (DIL), and verapamil (VER), under UV (254 nm) irradiation. The chemical structures of transformation byproducts (TBPs) were first identified to assess the potential ecological hazards. On that basis, a generic solid-phase extraction method, which simultaneously used four different cartridges, was adopted to extract and enrich the TBPs. Thereafter, the photo-degradation of target CCBs was performed under UV fluences typical for WWTP effluent disinfection. The degradation of all three CCBs conformed to the pseudo-firstorder kinetics, with rate constants of $0.031,0.044$ and $0.011 \mathrm{~min}^{-1}$ for AML, DIL and VER, respectively. By comparing the $\mathrm{MS}^{2}$ fragments and the evolution (i.e., formation or decay) trends of identified TBPs, the degradation pathways were proposed. In the WWTP effluent, although the target CCBs could be degraded, several TBPs still contained the functional pharmacophores and reached peak concentrations under UV fluences of $40-100 \mathrm{~mJ} \mathrm{~cm}^{-2}$.
\end{abstract}

(c) 2015 Elsevier Ltd. All rights reserved.

\section{Introduction}

In the last few decades, the occurrence of pharmaceuticals and personal care products (PPCPs) in the environment has received increasing attention and induces great challenge to the safety of aquatic ecosystems (Schwarzenbach et al., 2006). These PPCPs are mainly discharged from private households, hospitals and pharmaceutical industries, and eventually reach wastewater treatment plants (WWTPs). However, most of these compounds are only partly removed during wastewater treatment and thus appear in receiving waters and bio-solids (Batt et al., 2008; Chari and Halden, 2012).

\footnotetext{
* Corresponding authors.

E-mail addresses: carmesans@ub.edu (C. Sans), qiangz@rcees.ac.cn (Z. Qiang).
}

Calcium channel blockers (CCBs), such as amlodipine (AML), diltiazem (DIL), and verapamil (VER), are a group of pharmaceuticals that can selectively block the influx of calcium ions through a calcium channel. They are widely prescribed to lower the blood pressure and to treat arrythmias, angina and other heart diseases (Elliott and Ram, 2011). The occurrence of these CCBs in the WWTP effluents and downstream river waters have been frequently reported in recent years, with a concentration range of 3.0-510.0 ng $\mathrm{L}^{-1}$ (Hummel et al., 2006; Batt et al., 2008; Spongberg and Witter, 2008). Although no direct harmful effects to human have been reported, it is proved that an exposure to a certain concentration of CCBs in the WWTP effluent can induce ecological hazards to fish (Du et al., 2014). Wang and Gardinali (2013) also reported that DIL in reclaimed water can be ingested by fish and accumulate in fish body with a bio-concentration factor larger than 16 and an in-vivo half-life larger than $117 \mathrm{~h}$, indicating a potential ecological risk. 
Because of its distinct advantages such as high inactivation efficiency, easy operation, and small space-occupancy, ultraviolet (UV) technologies have been increasingly applied for drinking water and wastewater disinfection (Parkinson et al., 2001; Li and Blatchley, 2009). Recently, UV photolysis of water-borne PPCPs during the WWTP effluent disinfection process has been more frequently reported (Sun et al., 2014; Wang et al., 2014); however, few studies have focused on the degradation kinetics and mechanism of CCBs under UV irradiation. As revealed by previous researches, most CCBs (e.g., ALP, DIL) are unstable against UVA (320-400 nm), UVB (290-320 nm), or solar irradiation (Fasani et al., 2008; Kawabe et al., 2008), which indicates that the photodegradation of CCBs under $254 \mathrm{~nm}$ UV is possible.

Moreover, co-existing matrix materials, such as dissolved organic matter (DOM) and $\mathrm{NO}_{3}^{-}$, may also affect the degradation kinetics of micro-pollutants under UV irradiation by altering UV light penetration and producing/scavenging hydroxyl radicals $(\cdot \mathrm{OH})$. Mack and Bolton (1999) suggested that in the presence of $\mathrm{NO}_{2}^{-}$and $\mathrm{NO}_{3}^{-}$in aqueous solution, $\mathrm{UV}$ irradiation could yield $\cdot \mathrm{OH}$ with a quantum yield $(\phi \cdot \mathrm{OH})$ of up to $9 \%$. Wols and Hofman-Caris (2012) reported that DOM could not only act as important scavengers of $\cdot \mathrm{OH}$ with rate constants of about $(2-8) \times 10^{8} \mathrm{M}^{-1} \mathrm{~s}^{-1}$ in surface water and wastewater, but also produce other radicals to indirectly degrade organic compounds. They also summarized the photochemical constants of over 100 organic compounds; however, none of the selected CCBs was included.

In the present study, three CCBs, including AML, DIL and VER, were selected on behalf of the 1,4-dihydropyridine, 1,5benzothiazepine and phenylalkylamine classes, respectively. The degradation kinetics was investigated and the transformation byproducts (TBPs) were identified after exposing a relatively high concentration level of studied CCBs (i.e., $1.0 \mathrm{mg} \mathrm{L}^{-1}$ each) to UV irradiation in Milli-Q water (MQ water). Then, the existence of functional pharmacophores in the TBP structures was examined and the degradation pathways were proposed accordingly. On that basis, a generic solid-phase extraction (SPE) method, which simultaneously used four different cartridges with different extraction modes, was adopted to extract and enrich TBPs formed in the photolytic experiments of target CCBs at a relatively low concentration level (i.e., $100 \mathrm{ng} \mathrm{L}^{-1}$ each) under UV fluences typical for WWTP effluent disinfection. Moreover, the effects of co-existing matrix materials, including humic acid (HA), $\mathrm{NO}_{3}^{-}$and $\mathrm{Cl}^{-}$, on the photo-degradation of AML were particularly clarified.

\section{Materials and methods}

\subsection{Chemicals}

The studied CCBs (>99\% purity) including (+/-) verapamil hydrochloride (CAS No. 152-11-4), amlodipine besylate (CAS No. 111470-99-6) and (+)-cis-diltiazem hydrochloride (CAS No. 3328622-5) were purchased from Sigma Aldrich (Schnelldorf, Germany), whose major physicochemical properties are shown in Table 1. Internal standards (amlodipine- $\mathrm{d}_{4}$ maleic acid and verapamil- $\mathrm{d}_{6}$ hydrochloride salt) were purchased from Toronto Research Chemicals (Ontario, Canada). Methanol and acetonitrile (ACN) were purchased from Fisher Scientific (Geel, Belgium), and ethyl acetate from Merck (Darmstadt, Germany). Formic acid (98\%-100\%, ACS grade) and HA sodium salt (CAS No. 68131-04-4, technical grade) were obtained from Sigma Aldrich, and $\mathrm{KNO}_{3}$ and $\mathrm{NaCl}$ were from Panreac (Barcelona, Spain).

\subsection{UV reaction system}

Photo-degradation experiments were conducted in batch mode with a $2.0 \mathrm{~L}$ cylindrical glass reactor. The reaction solution was magnetically stirred and maintained at a constant temperature of $25^{\circ} \mathrm{C}$ using a water bath (Fig. S1). A low-pressure mercury lamp (4 W, 30\% UVC efficiency, Philips TUV G4T5) was used as the light source, which was initially warmed up for about $10 \mathrm{~min}$ to ensure a relatively stable output prior to each experiment. Atrazine actinometry was applied to measure the average UV fluence rate in the reactor (Canonica et al., 2008), which was equal to $0.77 \mathrm{~mW} \mathrm{~cm}^{-2}$ for MQ water and $0.52 \mathrm{~mW} \mathrm{~cm}^{-2}$ for the WWTP effluent (Text S1).

\subsection{Experimental procedures and sample pretreatment}

Photolytic experiments were conducted at two concentration levels of the target CCBs. Each experiment was run in triplicate and all glass containers were covered with aluminum foils to avoid

Table 1

Major physicochemical properties of target CCBs.

\begin{tabular}{|c|c|c|c|c|c|c|}
\hline Compound & Structure & Chemical formula & $\begin{array}{l}\text { Molecular weight } \\
\left(\mathrm{g} \mathrm{mol}^{-1}\right)\end{array}$ & $\begin{array}{l}\text { Water solubility } \\
\left(\mathrm{mg} \mathrm{L}^{-1}\right)\end{array}$ & $\mathrm{pK}_{a}^{\mathrm{a}}$ & $\log K_{o w}$ \\
\hline $\begin{array}{l}\text { Amlodipine } \\
\text { (AML) }\end{array}$ & & $\mathrm{C}_{20} \mathrm{H}_{25} \mathrm{O}_{5} \mathrm{~N}_{2} \mathrm{Cl}$ & 408.1452 & 7.4 & 9.45 & 2.22 \\
\hline $\begin{array}{l}\text { Diltiazem } \\
\text { (DIL) }\end{array}$ & & $\mathrm{C}_{22} \mathrm{H}_{26} \mathrm{O}_{4} \mathrm{~N}_{2} \mathrm{~S}$ & 414.5178 & 16.8 & $8.18,12.86$ & 3.09 \\
\hline $\begin{array}{l}\text { Verapamil } \\
\text { (VER) }\end{array}$ & & $\mathrm{C}_{27} \mathrm{H}_{38} \mathrm{O}_{4} \mathrm{~N}_{2}$ & 454.6016 & 3.94 & 9.68 & 3.79 \\
\hline
\end{tabular}

a Calculated by the ALOGPS 2.1 software (http://www.vcclab.org). 
undesired photolysis. For the relatively high concentration level experiments, $1.0 \mathrm{mg} \mathrm{L}^{-1}$ AML, DIL, and VER aqueous solutions (prepared in MQ water) were irradiated individually to determine their degradation kinetics and identify their TBPs. The solution $\mathrm{pH}$ was controlled at 7.0 with $10 \mathrm{mM}$ phosphate buffer, so all the three CCBs were mainly present in the cationic form. At pre-selected time intervals, $1 \mathrm{~mL}$ aliquots of the irradiated sample were withdrawn and stored in $2 \mathrm{~mL}$ vials at $-20^{\circ} \mathrm{C}$ for later analysis.

For the relatively low concentration level experiments, the effluent collected from a local WWTP in Barcelona was used as matrix after being filtered through $0.7 \mu \mathrm{m}$ glass fiber filters, whose major quality parameters are given in Table S1. The studied CCBs were spiked together into the reaction solution with an initial concentration of $100 \mathrm{ng} \mathrm{L}^{-1}$ (each). At pre-selected time intervals, $400 \mathrm{~mL}$ aliquots of the irradiated sample were withdrawn, spiked with the internal standards ( $100 \mathrm{ng} \mathrm{L}^{-1}$ each), and extracted by the generic SPE method as described elsewhere (Zonja et al., 2015). In brief, samples were adjusted to $\mathrm{pH} 6.5 \pm 0.2$ and extracted simultaneously with four different cartridges: Oasis HLB $(500 \mathrm{mg} / 6 \mathrm{cc}$, Waters, Milford, USA), Bond Elut PPL (500 mg/6cc, Agilent, Waghaeusel-Wiesental, Germany), Oasis MAX and Oasis MCX (both $200 \mathrm{mg} / 6 \mathrm{cc}$, Waters). The HLB cartridge was selected for its broad capacity of enriching multiple classes of organic compounds, while the PPL, MAX and MCX cartridges were selected for their specialty of enriching weak-polar, acidic and alkaline organic compounds, respectively. The four cartridges were connected in series, and conditioned sequentially with $5 \mathrm{~mL}$ of methanol/ethyl acetate $(1: 1, \mathrm{v} / \mathrm{v})$ and $5 \mathrm{~mL}$ of MQ water. After an irradiated sample (400 mL) was extracted, the HLB and PPL cartridges were eluted with $3 \times 3 \mathrm{~mL}$ of methanol/ethyl acetate (1:1), and the MAX and MCX cartridges were eluted sequentially with $3 \mathrm{~mL}$ of methanol/ ethyl acetate (1:1) and $2 \times 3 \mathrm{~mL}$ of methanol with $2 \%$ formic acid (for MAX) or methanol with $5 \%$ ammonia (for MCX). The resulting four extracts were combined together, evaporated to dryness under a gentle stream of nitrogen gas, and reconstituted with $400 \mu \mathrm{L}$ of ACN/water (10:90) for later analysis. The generic SPE method could achieve acceptable recovery efficiencies for the target CCBs and identified TBPs (54.3\%-119.9\%, Table S2).

\subsection{Analytical methods}

The parent CCBs and their TBPs formed in photolysis experiments were detected using an Acquity ultra performance liquid chromatograph (UPLC, Waters) coupled to a Q-Exactive mass spectrometer (Thermo Scientific, Bremen, Germany), with an injection volume of $10 \mu \mathrm{L}$. Chromatographic separation was performed with an Acquity C18 column $(100 \times 2.1 \mathrm{~mm}, 1.7 \mu \mathrm{m})$ that was guarded by a pre-column of the same packing material $(5 \times 2.1 \mathrm{~mm}, 1.7 \mu \mathrm{m})$. Elution gradient with two mobile phases, including $\mathrm{ACN}$ with $0.1 \%$ formic acid $(\mathrm{A})$ and MQ water with $0.1 \%$ formic acid (B), were programmed as follows (min, \%A): $(0,10),(1$, $10),(5,90),(6,90),(6.5,10)$, and $(8,10)$. The flow rate was $0.3 \mathrm{~mL} \mathrm{~min}^{-1}$ and the column temperature was held constant at $40{ }^{\circ} \mathrm{C}$.

Electrospray ionization interface was operated in the positive ion mode with spray voltage of $+3.0 \mathrm{kV}$, heater temperature of
$250{ }^{\circ} \mathrm{C}$, and capillary temperature of $350{ }^{\circ} \mathrm{C}$. Data-dependent scan mode was applied for identification of TBPs with the following operation parameters: resolution of both full and $\mathrm{MS}^{2}$ scans $=35000$ (full width at half maximum), scan range $=50-600 \mathrm{~m} / \mathrm{z}$, isolation window $=2 \mathrm{~m} / \mathrm{z}$, and collision energy $=35 \mathrm{eV}$. The $\mathrm{MS}^{2}$ scan could confirm the structures of the parent compounds and TBPs.

\section{Results and discussion}

\subsection{Degradation kinetics of target CCBs in MQ water}

The UV degradation of three CCBs, with an initial concentration of $1.0 \mathrm{mg} \mathrm{L}^{-1}$, was conducted individually in MQ water at $\mathrm{pH} 7.0$. All experiments lasted for $60 \mathrm{~min}$, which corresponds to a UV fluence of $2772 \mathrm{~mJ} \mathrm{~cm}^{-2}$ (UV fluence rate $=0.77 \mathrm{~mW} \mathrm{~cm}^{-2}$, Text S1). The photolysis rate constants, photochemical parameters and removal efficiencies of target CCBs are shown in Table 2. The degradation of AML, DIL and VER followed the pseudo-first-order kinetics with time-based rate constants $\left(k_{\mathrm{d}}^{\prime}\right)$ of $0.031,0.044$, and $0.011 \mathrm{~min}^{-1}$, respectively. The molar absorption coefficients $\left(\varepsilon_{254}\right)$ of target CCBs were determined from their absorption spectra (Fig. S3), and then the quantum yields at $254 \mathrm{~nm}\left(\phi_{254}\right)$ could be calculated with Eq. (1) (Baeza and Knappe, 2011):

$\phi_{254}=\frac{k_{\mathrm{d}}^{\prime} \times U_{254}}{60 \times 0.77 \times \ln (10) \times \varepsilon_{254}}$

where $U_{254}$ is the molar photon energy at $254 \mathrm{~nm}\left(4.72 \times 10^{5} \mathrm{JE}^{-1}\right)$, and $60(\mathrm{~min})$ and $0.77\left(\mathrm{~mW} \mathrm{~cm}^{-2}\right)$ convert the time-based $k_{\mathrm{d}}^{\prime}$ to its fluence-based value. As shown in Table 2, the $\varepsilon_{254}$ of AML, DIL and VER was 37266, 39153 and $8042 \mathrm{M}^{-1} \mathrm{~cm}^{-1}$, respectively; and the $\phi_{254}$ was $0.0037,0.0050$ and $0.0061 \mathrm{~mol} \mathrm{E}^{-1}$, respectively. After UV irradiation for $60 \mathrm{~min}$, the removal efficiencies of AML and DIL could reach $84.7 \%$ and $93.6 \%$, respectively; but VER was only removed by $51.2 \%$. According to the acquired $k_{\mathrm{d}}^{\prime}$ values, the UV fluences required to remove $90 \%$ of AML, DIL and VER are thus calculated to be 3335,2091 and $9315 \mathrm{~mJ} \mathrm{~cm}^{-2}$, respectively, which are much higher than applied for wastewater disinfection $\left(40-100 \mathrm{~mJ} \mathrm{~cm}^{-2}\right)$.

\subsection{Identification of TBPs and proposed degradation pathways}

The major TBPs were also identified in the relatively high concentration level experiments. The full scan of Q-Exactive MS was first used to register the exact mass of each TBP and determine their evolution (i.e., formation or decay) trends by calculating their relative concentrations. Afterward, the $\mathrm{MS}^{2}$ scan was performed to obtain the fragmentation patterns of identified TBPs to elucidate their structures. This analytical approach is considered efficient in elucidating TBP structures when authentic standards are not available (Schymanski et al., 2014). Moreover, whether or not the functional pharmacophores existed in the TBP structures was also examined to assess the potential ecological hazards.

The major physicochemical properties and $\mathrm{MS}^{2}$ fragmentation patterns of the target CCBs and identified TBPs are shown in Table S3.

Table 2

Photolysis rate constants, photochemical parameters, and removal efficiencies of target CCBs (UV fluence $=2772 \mathrm{~mJ} \mathrm{~cm}{ }^{-2}, \mathrm{pH}^{2}=7.0$ ).

\begin{tabular}{|c|c|c|c|c|c|}
\hline Compound & $\begin{array}{l}\text { Photolysis rate constant, } \\
k_{\mathrm{d}}^{\prime} \times 10^{2}\left(\mathrm{~min}^{-1}\right)^{\mathrm{a}}\end{array}$ & $\mathrm{R}^{2}$ & $\begin{array}{l}\text { Molar absorption coefficient, } \\
\varepsilon_{254}\left(\mathrm{M}^{-1} \mathrm{~cm}^{-1}\right)^{\mathrm{a}}\end{array}$ & $\begin{array}{l}\text { Quantum yield, } \\
\phi_{254} \times 10^{3}\left(\mathrm{~mol} \mathrm{E}^{-1}\right)^{\mathrm{a}}\end{array}$ & Removal (\%) \\
\hline AML & $3.07 \pm 0.04$ & 0.990 & $37266 \pm 84$ & $3.68 \pm 0.11$ & 84.7 \\
\hline DIL & $4.43 \pm 0.31$ & 0.950 & $39153 \pm 42$ & $4.98 \pm 0.34$ & 93.6 \\
\hline VER & $1.11 \pm 0.03$ & 0.942 & $8042 \pm 40$ & $6.08 \pm 0.10$ & 51.2 \\
\hline
\end{tabular}

${ }^{\mathrm{a}}$ Mean \pm standard deviation $(n=3)$. 


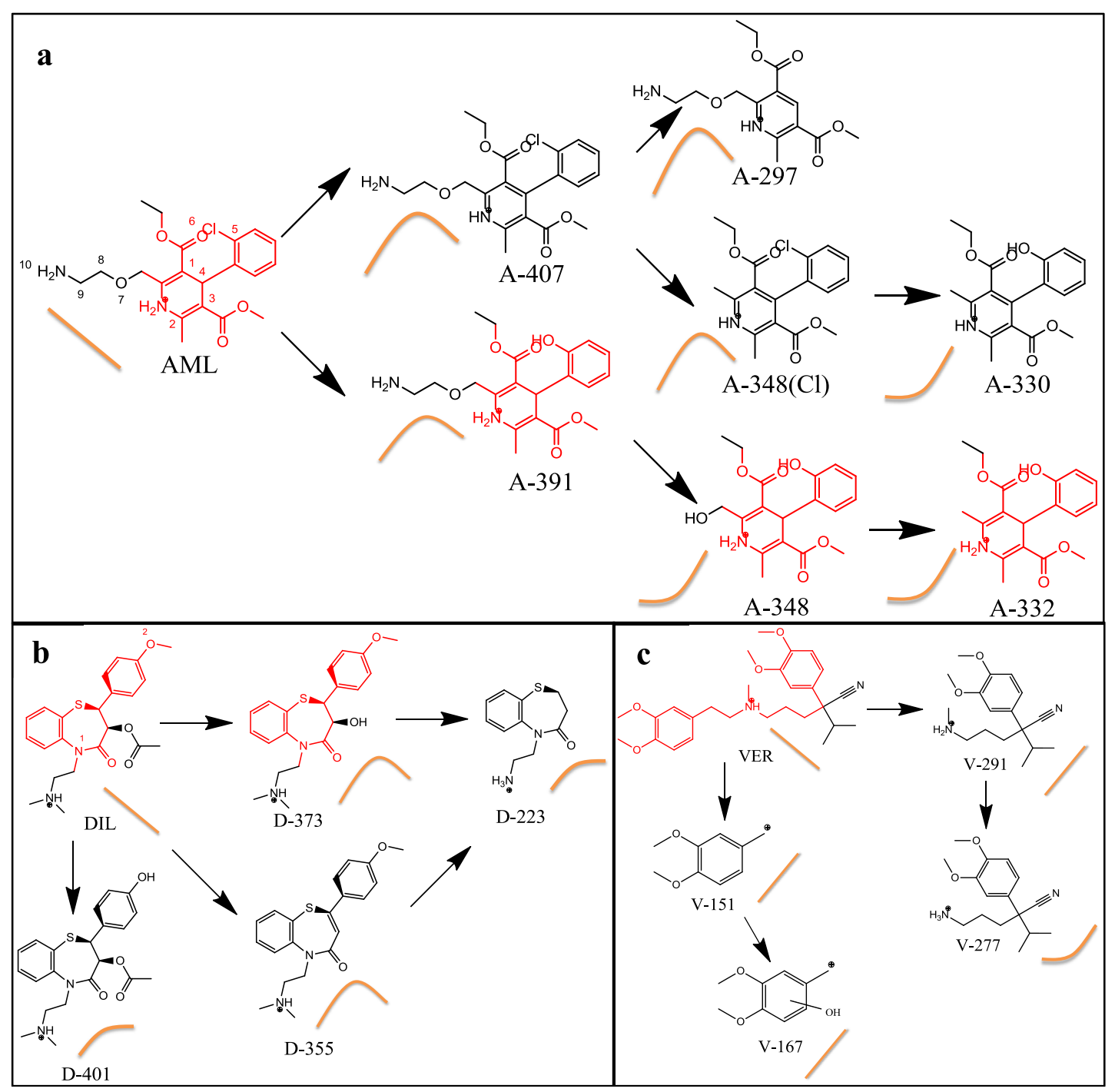

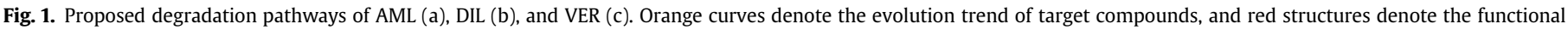
pharmacophores. (For interpretation of the references to color in this figure legend, the reader is referred to the web version of this article.)

Based on the evolution trends and chemical structures of identified TBPs, two degradation pathways of AML were proposed, which corresponded to two primary TBPs. One of the primary TBPs was amlodipine pyridine derivative (A-407, $\mathrm{m} / \mathrm{z}$ 407.1371), which was probably formed through a radical cation intermediate (Fasani et al., 2008). Under UV irradiation, the A-407 could lose the chlorophenyl group to form A-297 or lose the aminoethoxyl group to form A$348(\mathrm{Cl})$ (Fig. 1a). The $\mathrm{Cl}$ atom of $\mathrm{A}-348(\mathrm{Cl})$ could be further attacked by $\cdot \mathrm{OH}$ to form A-330. Jakimska et al. (2014) also detected the three TBPs (i.e., A-297, A-348(Cl), and A-330) during photolysis of AML with a xenon lamp (1000 W, 250-1000 nm) and classified them as the secondary byproducts. However, we found that A-330 only appeared after 15 min of UV irradiation, while A-348(Cl) appeared after $3 \mathrm{~min}$ and reached a peak concentration at $30 \mathrm{~min}$. Therefore, A-330 tended to originate from the degradation of A-348(Cl) and should be classified as a tertiary byproduct. The second degradation pathway started with the formation of A-391, which was probably attributed to the attack of $\cdot \mathrm{OH}$ on the phenol ring of AML. Then, A391 could sequentially produce A-348 and A-332 by losing the aminoethyl group and further losing the hydroxyl group. It is noted that none of the TBPs in the second pathway has been reported previously. This is most likely because the former study was performed in river water (Jakimska et al., 2014), so the matrix materials could strongly compete for $\cdot \mathrm{OH}$ and inhibit the formation of A-391. The inhibition on A-391 formation by co-existing matrix materials was also observed in this study (see Section 3.4).

Moreover, the two degradation pathways showed different elimination efficiencies of the functional pharmacophore (highlighted in red). In the first pathway, when A-407 was formed, the aromatization of the 1,4-dihydropiridine moiety could significantly alter the original steric structure of AML by rotating the chlorophenyl group around Site 4 (Fig. 1a) to form the delocalized $\pi$ bond between the pyridine and phenol rings. As a result, the boatform structure of 1,4-dihydropiridine, which is crucial for the receptor protein (Rojstaczer and Triggle, 1996), was destructed. By contrast, the pharmacophore persisted in the TBPs of the second (A-391) pathway because either the substitution of $\mathrm{Cl}$ with $\mathrm{OH}$ at Site 5 or the rupture of the aliphatic chain at Sites $7-10$ could hardly reduce the therapeutic activity of 1,4-dihydropiridine pharmaceuticals (Yamamoto et al., 2006; Reimao et al., 2010). Therefore, the first pathway is more efficient in eliminating the ecological hazards related to AML.

In the degradation of DIL, a total of four TBPs were identified, including D-373 (by losing the acetyl group), D-355 (by losing the 
acetoxy group and forming a double bond), D401 (by substituting $\mathrm{CH}_{3}$ with $\mathrm{H}$ at Site 2), and D-223 (by losing the acetyl and methoxyphenyl groups), all of which appeared quickly after 1 min of UV irradiation. D-373 and D-355 reached their peak concentrations at $15 \mathrm{~min}$ and started to decay afterward, while D-401 and D223 continuously accumulated to reach a nearly stable concentration (Fig. 1b). By comparing their structures, D-373, D-355 and D401 were classified as the primary TBPs, while D-223, which tended to originate from the degradation of D-373 and D-355, was classified as the secondary TBP. A previous study on the structure-activity relationship has revealed that the tertiary amino (Site 1) and $p$-methoxy (Site 2) groups are responsible for receptor bonding, while the loss of either an acetyl or a methyl (attached to the amino cation) group has little impact on the therapeutic activity (Li et al., 1992). Thus, D-401 and D-223 were likely to lose the therapeutic activity, while D-373 was likely maintain it. In regard to D-355, the formation of a double bond in the heterocyclic ring may severely alter its steric structure and thus destroy the therapeutic activity. Compared with D-373 and D401, D-355 could no longer form the $\mathrm{MS}^{2}$ fragment of $\mathrm{m} / \mathrm{z} 178$, which implies the destruction of its original steric structure (Table S3).

The degradation pathway of VER started with the bond cleavage between the methyl and the cationic amino groups (Fig. 1c), leading to the formation of two primary TBPs (V-151 and V-291) whose concentrations continuously increased during the reaction. Furthermore, V-167 could be produced when the phenol ring of V151 was attacked by $\cdot \mathrm{OH}$ and $\mathrm{V}-277$ could be produced when $\mathrm{V}-291$ lost a methyl group attached to the cationic amino group. In UV photolysis, V-277 only appeared after $10 \mathrm{~min}$ and had similar $\mathrm{MS}^{2}$ fragments to V-291. Because the cationic amino group and the two phenol rings all account for the therapeutic activity (Mannhold et al., 1978; Toffoli et al., 1995), the four TBPs are free of ecological hazards.

\subsection{Degradation of target CCBs in WWTP effluent}

To simulate wastewater disinfection process, a low concentration level of target CCBs (100 $\mathrm{ng} \mathrm{L}^{-1}$ each) and a typical UV fluence range $\left(0-230 \mathrm{~mJ} \mathrm{~cm}^{-2}\right)$ were selected for photolytic experiments in the WWTP effluent. The degradation kinetics of target CCBs in MQ water and WWTP effluent are compared in Fig. 2. In the WWTP effluent, the degradation of AML and VER was obviously promoted. Specifically, the removal efficiency of VER was about 4 times that in MQ water at the end of the reaction (UV fluence $=230 \mathrm{~mJ} \mathrm{~cm}{ }^{-2}$ ), while the concentration of AML was below its detection limit after $40 \mathrm{~mJ} \mathrm{~cm}^{-2}$. This result agrees with other previous works. Jakimska et al. (2014) reported that the degradation of AML was promoted in river water and WWTP influent/effluent under the irradiation of a xenon lamp, compared to that in MQ water. Giri et al. (2014) suggested that the organic materials in the WWTP effluent should have triggered indirect photolysis to enhance the degradation of AML under UV irradiation. However, Kim et al. (2009) reported that the WWTP effluent could have different impacts (either promotion or inhibition) on the UV photolysis of different PPCPs. In the present study, we also found that the degradation of DIL in the WWTP effluent was significantly inhibited in the later phase of the reaction.

The background concentrations of three studied CCBs and their TBPs in the raw effluent were also determined. Table 3 shows that AML, DIL and VER had a background concentration of 2.5, 93.2 and $13.6 \mathrm{ng} \mathrm{L}^{-1}$, respectively. Previous researches have also reported the occurrence of these CCBs in WWTP effluents (Batt et al., 2008; Spongberg and Witter, 2008; Tarcomnicu et al., 2011). In addition, the two primary TBPs (A-407 and A-391) of AML were also detected in the raw effluent. As mentioned above, A-407 could be generated from the degradation of AML under UV or solar irradiation (Fasani et al., 2008; Jakimska et al., 2014), and A-391 could be generated by an attack of $\bullet \mathrm{OH}$ on the $\mathrm{Cl}$ atom. Therefore, their occurrence in the raw effluent might result from solar irradiation and/or biological transformation.

The relative concentration was used to depict the evolution (formation or decay) trends of the identified TBPs (Table 3). For a target TBP, the relative concentration was calculated by dividing a selected peak area by the maximum peak area detected during the whole reaction course (the background peak area was subtracted if this TBP was already detected in the raw effluent). Results indicate that although AML was completely removed after $40 \mathrm{~mJ} \mathrm{~cm}^{-2}$, the two primary TBPs (i.e., A-391 and A-407) reached their maximum concentrations at 40 and $80 \mathrm{~mJ} \mathrm{~cm}^{-2}$, respectively. Moreover, the formation of A-391 reflected the generation of $\cdot \mathrm{OH}$ in the UV irradiated solution. At the UV fluence of $160 \mathrm{~mJ} \mathrm{~cm}^{-2}, \mathrm{~A}-407$ achieved a total removal, but $58.3 \%$ of A-391 remained in the solution. Because A-391 contains the functional pharmacophore (Fig. 1), the treated solution was still ecologically hazardous. As for DIL, the three primary TBPs (D-355, D-373 and D-401) reached their maximum concentrations at 40,40 and $80 \mathrm{~mJ} \mathrm{~cm}^{-2}$, respectively. Afterward, because the degradation of DIL was inhibited, no more TBPs were formed and their concentrations started to decrease. At the UV fluence of $160 \mathrm{~mJ} \mathrm{~cm}^{-2}$, D-355 and D-401 were completely removed, while $68.4 \%$ of $\mathrm{D}-373$, which contains the functional pharmacophore (Fig. 1), still remained in the treated solution. As for VR, the two primary TBPs (V-151 and V-291) exhibited a different

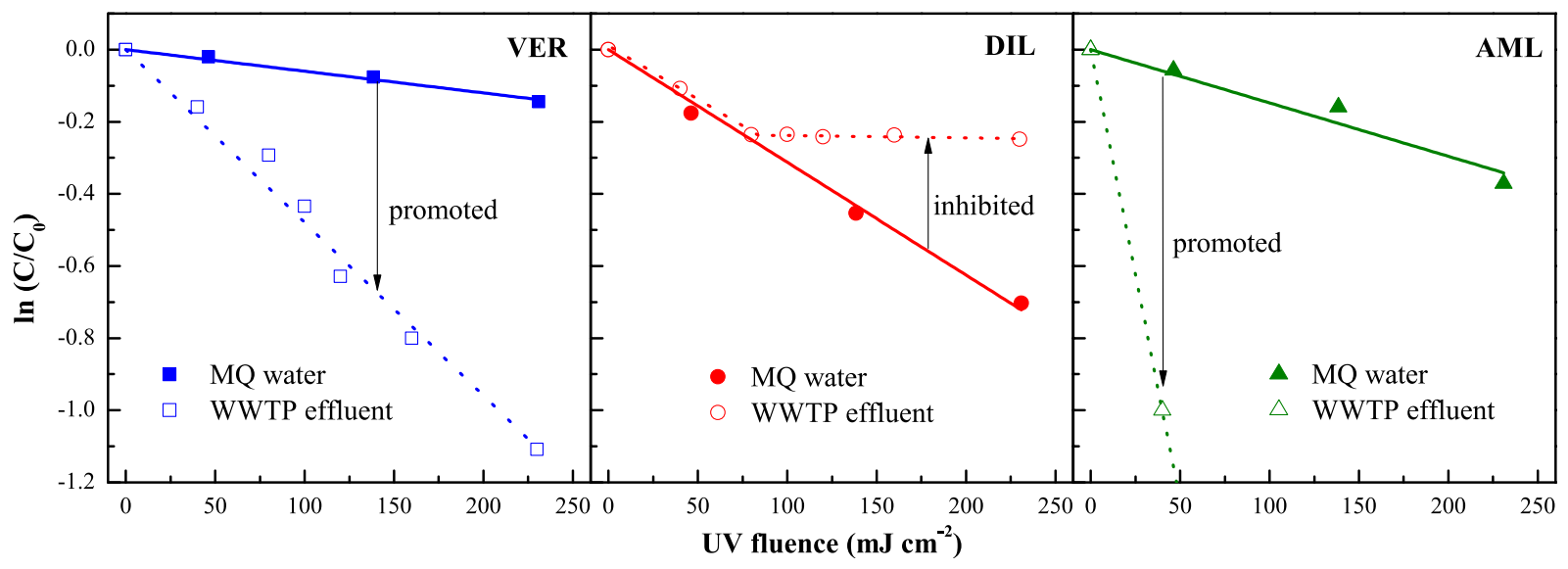

Fig. 2. Degradation of target CCBs under UV irradiation in MQ water and WWTP effluent. 
Table 3

Relative concentrations (\%) of target CCBs and their primary TBPs under UV irradiation in WWTP effluent.

\begin{tabular}{|c|c|c|c|c|c|c|c|c|c|c|}
\hline \multirow{2}{*}{$\begin{array}{l}\text { UV fluence } \\
\left(\mathrm{mJ} \mathrm{cm}{ }^{-2}\right)\end{array}$} & \multirow[t]{2}{*}{ AML } & \multicolumn{2}{|c|}{ Primary TBPs } & \multirow[t]{2}{*}{ DIL } & \multicolumn{3}{|c|}{ Primary TBPs } & \multirow[t]{2}{*}{ VER } & \multicolumn{2}{|c|}{ Primary TBPs } \\
\hline & & A-391 & A-407 & & D-355 & D-373 & D-401 & & V-151 & V-291 \\
\hline 0 & 100.0 & 0.0 & 0.0 & 100.0 & 0.0 & 0.0 & 0.0 & 100.0 & 0.0 & 0.0 \\
\hline 40 & 0.0 & 100.0 & 68.2 & 89.8 & 100.0 & 100.0 & 35.1 & 85.3 & 25.4 & 57.2 \\
\hline 80 & 0.0 & 88.8 & 100.0 & 79.0 & 95.4 & 85.5 & 100.0 & 77.6 & 100.0 & 100.0 \\
\hline 100 & 0.0 & 79.1 & 38.8 & 79.0 & 14.1 & 83.4 & 17.5 & 67.7 & 72.6 & 44.8 \\
\hline 120 & 0.0 & 76.3 & 1.7 & 73.5 & 0.0 & 76.3 & 0.0 & 53.3 & 62.6 & 11.0 \\
\hline 160 & 0.0 & 58.3 & 0.0 & 74.3 & 0.0 & 68.4 & 0.0 & 44.9 & 38.6 & 0.0 \\
\hline $\mathrm{C}_{\mathrm{BG}}{ }^{\mathrm{a}}$ & 2.5 & 3.7 & 4.1 & 93.2 & 0.0 & 0.0 & 0.0 & 13.6 & 0.0 & 0.0 \\
\hline Risk $^{\mathrm{b}}$ & + & + & - & + & + & + & - & + & - & - \\
\hline
\end{tabular}

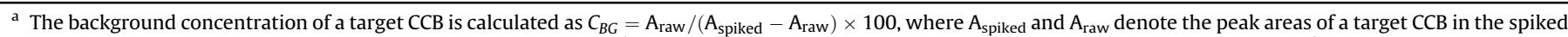

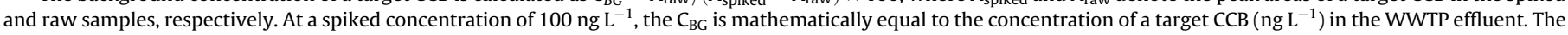
background concentrations of TBPs are calculated in the same way, except that $\mathrm{A}_{\text {spiked }}$ is replaced by $\mathrm{A}_{100 \%}$ (i.e., the largest detected peak area).

$\mathrm{b}$ Risk is assessed based on whether or not a target analyte contains the functional pharmacophore.

evolution trend in the WWTP effluent, as compared to their unceasing accumulation in MQ water (Fig. 1). Both V-291 and V-151 reached their peak concentrations at $80 \mathrm{~mJ} \mathrm{~cm}^{-2}$ and then started to decrease, implying that the matrix materials may induce some further degradation pathways. Although $38.6 \%$ of V-151 remained at the UV fluence of $160 \mathrm{~mJ} \mathrm{~cm}^{-2}$, the treated solution was free of ecological hazards because V-151 does not contain the functional pharmacophore. In summary, all of the identified primary TBPs reached their maximum concentrations at a UV fluence ranging from 40 to $100 \mathrm{~mJ} \mathrm{~cm}{ }^{-2}$, which is typical for disinfection of wastewater and reclaimed water (according to the EU guideline, $40-80 \mathrm{~mJ} \mathrm{~cm}^{-2}$ for wastewater and $100 \mathrm{~mJ} \mathrm{~cm}^{-2}$ for reclaimed water). It is seen that the ecological hazards of AML and DIL cannot be completely eliminated in the UV disinfection process.

\subsection{Impacts of co-existing materials and $\mathrm{pH}$ on $\mathrm{AML}$ degradation}

To explore the possible factors affecting the degradation of AML, a series of photolytic experiments was carried out in MQ water under typical $\mathrm{pH}$ conditions or with externally spiked $\mathrm{Cl}^{-}$ (1600 $\left.\mathrm{mg} \mathrm{L}^{-1}\right)$, HA (50 mg L ${ }^{-1}$ as TOC) and $\mathrm{NO}_{3}^{-}\left(48 \mathrm{mg} \mathrm{L}^{-1}\right)$ (Table S4). The degradation of AML and the evolution of detected TBPs (A-391, A-407 and A-330) were examined, as illustrated in Fig. 3.

Fig. 3a shows that AML was degraded more quickly at 8.5 than at 7.0. The $\phi_{254}$ of AML, which denotes the efficiency of photolysis, was calculated to be 0.0037 and $0.0098 \mathrm{~mol} \mathrm{E}^{-1}$ at $\mathrm{pH}$ values of 7.0 and 8.5 , respectively, based on its $\varepsilon_{254}$ (Fig. S3). The change of $\mathrm{pH}$ could affect the speciation of AML in the reaction solution and subsequently alter its reactivity under UV irradiation. Baeza and Knappe (2011) also found that the $\phi_{254}$ increased with increasing $\mathrm{pH}$ for the UV photolysis of trimethoprim. Correspondingly, A-391 was generated more quickly at pH 8.5 (Fig. 3b). However, the formation of A-407 was a little slower at pH 8.5 (Fig. 3c), probably because it was degraded faster to produce A-330 (Fig. 3d). A higher pH seemed to facilitate the substitution of $\mathrm{Cl}$ with $\mathrm{OH}$ on the phenol ring.

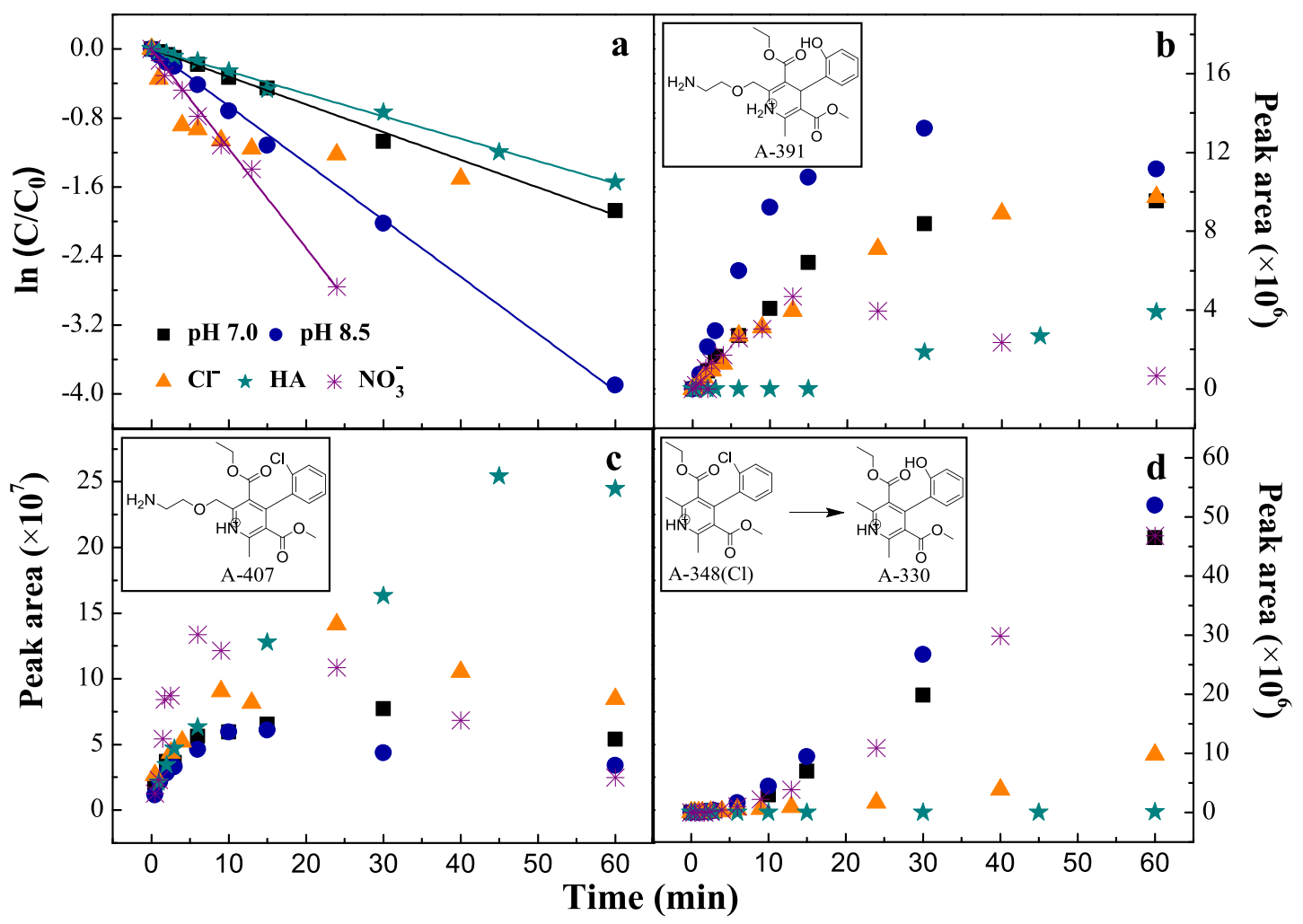

Fig. 3. Degradation of AML (a) and evolution of identified TBPs including A-391 (b), A-407 (c), and A-330 (d) under different experimental conditions. 
In the presence of $\mathrm{Cl}^{-}$and at $\mathrm{pH} 7.0$, the degradation of AML was the fastest in the first $4 \mathrm{~min}$ and then slowed down remarkably (Fig. 3a). It is well known that UV irradiation of $\mathrm{Cl}^{-}$produces $\mathrm{Cl} \cdot$, which can easily react with organic compounds. As the evolution trends of A-391 and A-407 are similar to those in MQ water at $\mathrm{pH} 7.0$ (Fig. 3b and c), the initially promoted degradation of AML was probably attributed to the formation of chlorinated compounds. Results also indicate that the concentration of A-330 started to increase slowly after $24 \mathrm{~min}$ and was much lower than that in MQ water (Fig. 3d). Considering that A-391 continuously accumulated in the solution over the whole reaction course, A-330 could only originate from the degradation of A-407. This result partly confirms the degradation pathways of AML as proposed in Fig. 1a.

In the presence of $\mathrm{HA}$ and at an initial $\mathrm{pH}$ of 5.9, the degradation of AML was inhibited to some extent (Fig. 3a). Because HA strongly competed for $\cdot \mathrm{OH}$ (Wols and Hofman-Caris, 2012), the formation of A-391 and A-330, which was triggered by an attack of $\cdot \mathrm{OH}$ on the $\mathrm{Cl}$ atom, was obviously inhibited (Fig. $3 \mathrm{~b}$ and d). Consequently, the inhibited formation of A-330 led to the continuous accumulation of A-407 during most of the reaction course (Fig. 3c). Therefore, the A407 pathway predominated in the degradation of AML when a high concentration of HA was available.

Among all tested influential factors, $\mathrm{NO}_{3}^{-}$showed the greatest promotion on the degradation of AML (Fig. 3a). Along with the degradation of AML, A-391 and A-407 were formed quickly in the beginning of the reaction and then decayed gradually (Fig. $3 \mathrm{~b}$ and c), while A-330 unceasingly accumulated over the whole reaction course. As UV irradiation of $\mathrm{NO}_{3}^{-}$produces $\cdot \mathrm{OH}$ with $\phi \cdot \mathrm{OH}$ of up to $9 \%$ (Mack and Bolton, 1999), the further degradation of A-391 and A407 could be ascribed to the indirect oxidation of $\bullet \mathrm{OH}$.

\section{Conclusions}

In this study, the degradation kinetics and pathways of three CCBs under UV irradiation were investigated in both MQ water and WWTP effluent. In addition, the elimination of functional pharmacophores from the TBP structures was also examined to assess the potential ecological hazards. Based on the experimental results, the following conclusions can be drawn:

- UV photolysis of three studied CCBs conformed to the pseudofirst-order reaction kinetics, with rate constants of 0.031 , $0.044,0.011 \mathrm{~min}^{-1}$ for AML, DIL and VER, respectively.

- Several TBPs of AML and DIL still contained the functional pharmacophores, while those of VER were free of ecological hazards.

- The pharmacophore-containing TBPs of AML and DIL reached their peak concentrations under UV fluences typical for disinfection of wastewater and reclaimed water (i.e., $40-100 \mathrm{~mJ} \mathrm{~cm}^{-2}$ ), indicating potential ecological hazards.

- The degradation of VER and AML was promoted in the WWTP effluent, while the degradation of DIL was inhibited. The removal efficiencies of AML, DIL and VER in the WWTP effluent were $100 \%, 21 \%$ and $32 \%$ at $100 \mathrm{~mJ} \mathrm{~cm}^{-2}$, respectively.

- An increase in $\mathrm{pH}$ (from 7.0 to 8.5 ) or the presence of $\mathrm{NO}_{3}^{-}$ promoted the degradation of AML, while the presence of HA exerted an inhibiting effect. In the presence of $\mathrm{Cl}^{-}$, the degradation of AML was the fastest in the beginning of the reaction and remarkably slowed down afterward.

\section{Acknowledgment}

This work was financially supported by the People Program (Marie Curie Actions) of the European Union's Seventh Program
FP7/2007-2013 under a REA grant (Agreement No. 318926) and the National Natural Science Foundation of China (51221892, 51138009). B. Zonja acknowledges the Marie Curie Actions ITN CSI: Environment PITN-GA-2010-264329 for the early-stage researcher contract and funding. S. Pérez acknowledges the contract from the Ramon y Cajal Program of the Spanish Ministry of Economy and Competitiveness. This work was also supported by the Generalitat de Catalunya (Consolidated Research Groups “2014 SGR 418-Water and Soil Quality Unit”, 2014 SGR 291-ICRA).

\section{Appendix A. Supplementary data}

Supplementary data related to this article can be found at http:// dx.doi.org/10.1016/j.watres.2015.05.028.

\section{References}

Baeza, C., Knappe, D.R.U., 2011. Transformation kinetics of biochemically active compounds in low-pressure UV photolysis and $\mathrm{UV} / \mathrm{H}_{2} \mathrm{O}_{2}$ advanced oxidation processes. Water Res. 45 (15), 4531-4543.

Batt, A.L., Kostich, M.S., Lazorchak, J.M., 2008. Analysis of ecologically relevant pharmaceuticals in wastewater and surface water using selective solid-phase extraction and UPLC-MS/MS. Anal. Chem. 80 (13), 5021-5030.

Canonica, S., Meunier, L., von Gunten, U., 2008. Phototransformation of selected pharmaceuticals during UV treatment of drinking water. Water Res. 42 (1-2), 121-128.

Chari, B.P., Halden, R.U., 2012. Validation of mega composite sampling and nationwide mass inventories for 26 previously unmonitored contaminants in archived biosolids from the U.S National Biosolids Repository. Water Res. 46 (15), 4814-4824.

Du, B.W., Price, A.E., Scott, W.C., Kristofco, L.A., Ramirez, A.J., Chambliss, C.K., Yelderman, J.C., Brooks, B.W., 2014. Comparison of contaminants of emerging concern removal, discharge, and water quality hazards among centralized and on-site wastewater treatment system effluents receiving common wastewater influent. Sci. Total Environ. 466, 976-984.

Elliott, W.J., Ram, C.V.S., 2011. Calcium channel blockers. J. Clin. Hypertens. 13 (9), 687-689.

Fasani, E., Albini, A., Gemme, S., 2008. Mechanism of the photochemical degradation of amlodipine. Int. J. Pharm. 352 (1-2), 197-201.

Giri, R.R., Ozaki, H., Guo, X., Takanami, R., Taniguchi, S., 2014. Significance of water quality and radiation wavelength for UV photolysis of PhCs in simulated mixed solutions. Cent. Eur. J. Chem. 12 (6), 659-671.

Hummel, D., Loffler, D., Fink, G., Ternes, T.A., 2006. Simultaneous determination of psychoactive drugs and their metabolites in aqueous matrices by liquid chromatography mass spectrometry. Environ. Sci. Technol. 40 (23), 7321-7328.

Jakimska, A., Sliwka-Kaszynska, M., Nagorski, P., Namiesnik, J., Kot-Wasik, A., 2014. Phototransformation of amlodipine: degradation kinetics and identification of its photoproducts. Plos One 9 (10), 1-10 (e109206).

Kawabe, Y., Nakamura, H., Hino, E., Suzuki, S., 2008. Photochemical stabilities of some dihydropyridine calcium-channel blockers in powdered pharmaceutical tablets. J. Pharm. Biomed. 47 (3), 618-624.

Kim, I., Yamashita, N., Tanaka, H., 2009. Photodegradation of pharmaceuticals and personal care products during UV and $\mathrm{UV} / \mathrm{H}_{2} \mathrm{O}_{2}$ treatments. Chemosphere 77 (4), 518-525.

Li, J., Blatchley, E.R., 2009. UV photodegradation of inorganic chloramines. Environ. Sci. Technol. 43 (1), 60-65.

Li, R.S., Farmer, P.S., Xie, M., Quilliam, M.A., Pleasance, S., Howlett, S.E., Yeung, P.K.F., 1992. Synthesis, characterization, and $\mathrm{Ca}^{2+}$ antagonistic activity of diltiazem metabolites. J. Med. Chem. 35 (17), 3246-3253.

Mack, J., Bolton, J.R., 1999. Photochemistry of nitrite and nitrate in aqueous solution: a review. J. Photochem. Photobiol. A 128 (1-3), 1-13.

Mannhold, R., Steiner, R., Haas, W., Kaufmann, R., 1978. Investigations on structureactivity-relationships of verapamil. N-S Arch. Pharmacol. 302 (2), 217-226.

Parkinson, A., Barry, M.J., Roddick, F.A., Hobday, M.D., 2001. Preliminary toxicity assessment of water after treatment with UV-irradiation and $\mathrm{UVC} / \mathrm{H}_{2} \mathrm{O}_{2}$. Water Res. 35 (15), 3656-3664.

Reimao, J.Q., Scotti, M.T., Tempone, A.G., 2010. Anti-leishmanial and antitrypanosomal activities of 1,4-dihydropyridines: in vitro evaluation and structure-activity relationship study. Bioorgan. Med. Chem. 18 (22), 8044-8053.

Rojstaczer, N., Triggle, D.J., 1996. Structure-function relationships of calcium antagonists. Biochem. Pharmacol. 51 (2), 141-150.

Schwarzenbach, R.P., Escher, B.I., Fenner, K., Hofstetter, T.B., Johnson, C.A., von Gunten, U., Wehrli, B., 2006. The challenge of micropollutants in aquatic systems. Science 313 (5790), 1072-1077.

Schymanski, E.L., Jeon, J., Gulde, R., Fenner, K., Ruff, M., Singer, H.P., Hollender, J., 2014. Identifying small molecules via high resolution mass spectrometry: communicating confidence. Environ. Sci. Technol. 48 (4), 2097-2098.

Spongberg, A.L., Witter, J.D., 2008. Pharmaceutical compounds in the wastewater process stream in Northwest Ohio. Sci. Total Environ. 397 (1-3), 148-157.

Sun, Q., Lv, M., Hu, A.Y., Yang, X.Y., Yu, C.P., 2014. Seasonal variation in the 
occurrence and removal of pharmaceuticals and personal care products in a wastewater treatment plant in Xiamen, China. J. Hazard Mater. 277, 69-75.

Tarcomnicu, I., van Nuijs, A.L.N., Simons, W., Bervoets, L., Blust, R., Jorens, P.G., Neels, H., Covaci, A., 2011. Simultaneous determination of 15 top-prescribed pharmaceuticals and their metabolites in influent wastewater by reversedphase liquid chromatography coupled to tandem mass spectrometry. Talanta 83 (3), 795-803.

Toffoli, G., Simone, F., Corona, G., Raschack, M., Cappelletto, B., Gigante, M., Boiocchi, M., 1995. Structure-activity relationship of verapamil analogs and reversal of multidrug-resistance. Biochem. Pharmacol. 50 (8), 1245-1255.

Wang, D., Sui, Q., Lu, S.G., Zhao, W.T., Qiu, Z.F., Miao, Z.W., Yu, G., 2014. Occurrence and removal of six pharmaceuticals and personal care products in a wastewater treatment plant employing anaerobic/anoxic/aerobic and UV processes in Shanghai, China. Environ. Sci. Pollut. Res. 21 (6), 4276-4285.
Wang, J., Gardinali, P.R., 2013. Uptake and depuration of pharmaceuticals in reclaimed water by mosquito fish (Gambusia holbrooki): a worst-case, multiple-exposure scenario. Environ. Toxicol. Chem. 32 (8), 1752-1758.

Wols, B.A., Hofman-Caris, C.H.M., 2012. Review of photochemical reaction constants of organic micropollutants required for UV advanced oxidation processes in water. Water Res. 46 (9), 2815-2827.

Yamamoto, T., Niwa, S., Ohno, S., Onishi, T., Matsueda, H., Koganei, H., Uneyama, H., Fujita, S.I., Takeda, T., Kito, M., Ono, Y., Saitou, Y., Takahara, A., Iwata, S., Shoji, M. 2006. Structure-activity relationship study of 1,4-dihydropyridine derivatives blocking N-type calcium channels. Bioorg. Med. Chem. Lett. 16 (4), 798-802.

Zonja, B., Delgado, A., Perez, S., Barcelo, D., 2015. LC-HRMS suspect screening for detection-based prioritization of iodinated contrast media photodegradates in surface waters. Environ. Sci. Technol. 49 (6), 3464-3472. 